\title{
Periaortic Pedicle Screw Removal with Endovascular Control of the Aorta and Intraoperative Aortography: A Case Report and Review of the Literature
}

\author{
Michael Q. Potter ${ }^{1} \quad$ Brandon D. Lawrence ${ }^{1} \quad$ Daniel V. Kinikini $^{2} \quad$ Darrel S. Brodke ${ }^{1}$ \\ ${ }^{1}$ Department of Orthopaedics, University of Utah, Salt Lake City, Utah, \\ United States \\ 2 Division of Vascular Surgery, University of Utah, Salt Lake City, Utah, \\ Address for correspondence Brandon D. Lawrence, MD, Department \\ of Orthopaedics, University of Utah, 590 Wakara Way, Salt Lake City, \\ UT 84108, United States (e-mail: brandon.lawrence@hsc.utah.edu).
} United States

Evid Based Spine Care J 2013;4:149-153.

\author{
Abstract \\ Keywords \\ - pedicle screw fixation \\ - vascular injury \\ - endovascular aortic \\ repair \\ - aortography \\ - aortic graft \\ - complication
}

Study Design Case report and review of the literature.

Objective The objective of the article is to report the case of a 20-year-old patient with a threatened aortic injury from pedicle screw instrumentation successfully managed without aortic grafting.

Methods The patient's clinical course is retrospectively reviewed. The offending hardware was removed after gaining endovascular control of the aorta.

Results Intraoperative aortography was normal and no graft was placed. The patient remains asymptomatic at 2 years after surgery.

Conclusions Hardware impinging on the aorta can safely be removed by gaining endovascular control of the aorta. In the setting of normal intraoperative aortography in a young patient, we recommend against further intervention to avoid the known morbidity of aortic grafting.

\section{Introduction}

Vascular injury is an uncommon but potentially devastating complication of spine surgery. Throughout its course, the aorta remains in close proximity to the thoracic and lumbar spine and is at risk from malpositioned pedicle screws or other hardware. ${ }^{1}$ Despite this risk, the reported rate of vascular injury from spine surgery is low. In one systematic review, Papadoulas et al found the incidence of vascular injury after excision of a herniated lumbar disc via a posterior approach to be less than $0.05 \%{ }^{2}$ In addition, Hicks et al systematically reviewed 14,570 pedicle screws placed in the thoracic and lumbar spine of 1,666 patients with adolescent idiopathic scoliosis (AIS); they found a $4.2 \%$ overall rate of screw malposition with 6 of 8,147 screws ( $0.07 \%$ ) abutting the aorta in the subgroup of studies that reported this finding. There were no reports of major vascular complications. ${ }^{3}$

Despite the low rates of vascular injury identified in these reviews, the literature contains multiple case reports in which pedicle screw instrumentation was associated with early or late aortic injuries. ${ }^{4-10}$ In the majority of these reports, treatment consisted of removing of the offending hardware with simultaneous open or endovascular aortic repair (EVAR). Here, we describe the case of an adolescent patient with a malpositioned pedicle screw abutting the thoracic aorta and new complaints of deep-seated thoracic pain. The screw was removed after gaining endovascular control of the aorta. Intraoperative aortography demonstrated normal flow in the thoracic aorta, so no graft was placed to avoid the known morbidity of EVAR. The relevant literature received

March 26, 2013

accepted

July 18, 2013 (c) 2013 Georg Thieme Verlag KG Stuttgart · New York
DOI http://dx.doi.org/ 10.1055/s-0033-1357358. ISSN 1663-7976. 
on management of threatened vascular injury after spine surgery is reviewed.

\section{Case Report}

The patient was a 20-year-old man who presented to clinic with 4 months of back pain. At the age of 15 years, he had undergone a T6-T8 posterior instrumented fusion with excision of an osteoblastoma from the right T7 pedicle and lamina. At that time, his primary symptom was midthoracic pain; this pain resolved within 3 months of his index surgery. When he returned to clinic 5 years later, he complained of deep-seated substernal and midthoracic pain. He denied paresthesias, weakness, or constitutional symptoms.

On physical examination, the patient had a well-healed midline incision with no signs of infection. He had normal strength, sensation, and reflexes in his bilateral upper and lower extremities. He had no incontinence of bowel or bladder. He had symmetric and easily palpable dorsalis pedis and radial pulses bilaterally.

Posteroanterior and lateral radiographs (-Fig. 1) and a computed tomographic (CT) scan of the thoracic spine (-Fig. 2) demonstrated that the left T6 screw penetrated the lateral cortex of the T6 pedicle and abutted the posteromedial aspect of the aortic wall. There was no pseudoaneurysm or periaortic inflammation identified to suggest frank penetration into the aortic lumen. However, it was felt that because of ongoing mechanical irritation by the abutting screw and the patient's new pain symptoms, there was an increased risk of aortic perforation, dissection, or thrombotic complication if the screw were left in place, and that therefore, it was advisable to remove it.

After a thorough discussion of the risks and benefits with the patient, his family, and the vascular surgery service, the patient elected to go to the operative theater with plans to obtain endovascular control of the aorta, remove the offend-

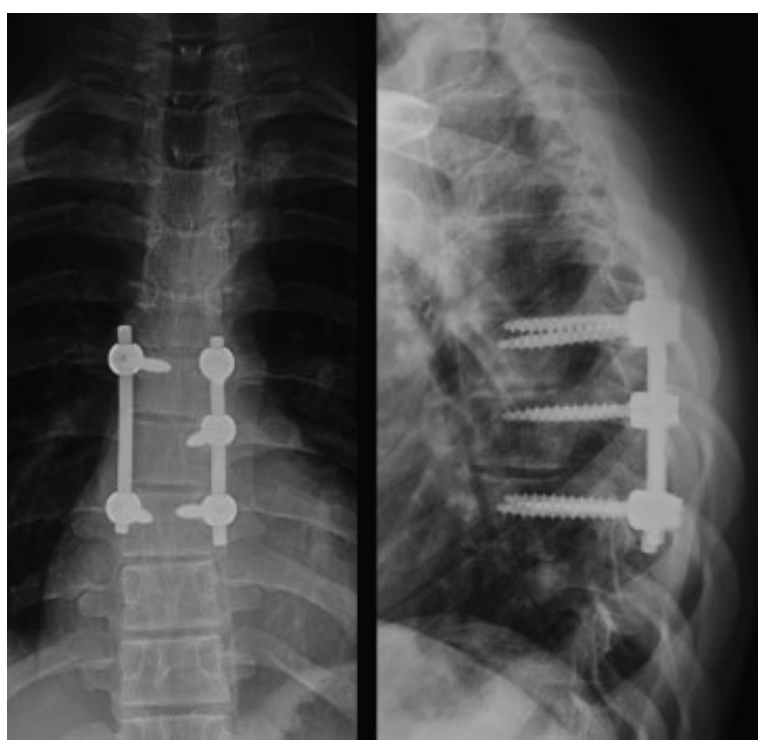

Fig. 1 Posteroanterior and lateral radiographs of the thoracic spine show lateral positioning of the left T6 pedicle screw.

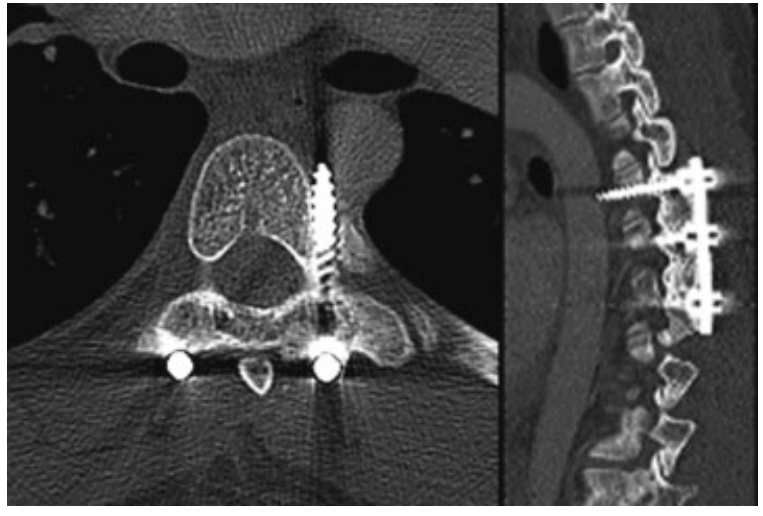

Fig. 2 An axial computed tomographic scan slice through the T6 pedicle and sagittal reconstruction show the left T6 pedicle screw breaches the lateral cortex and impinges against the posteromedial aorta.

ing hardware, and stent the aorta if required. In the theater, general anesthesia was induced and a double-lumen endotracheal tube was placed in case open thoracotomy were required. Antibiotics were administered before making a surgical incision. The patient was positioned supine and 7French sheath was introduced into the right common femoral artery. A catheter was passed via this sheath into the thoracic aorta and an aortogram was performed to confirm appropriate position of the catheter and adequate visualization of the aorta. No evidence of stenosis, contrast extravasation, or intraluminal material was noted.

With the right groin catheter in place, the patient was then moved and secured in the left lateral decubitus position. The previous midline thoracic incision was reopened and dissection was carried down to the level of the posterior instrumentation. The soft tissues were cleared from around the screws and the end caps removed. Once the vascular surgery team indicated their readiness to proceed, the left T6 screw was backed out. No significant bleeding was noted from the screw site. The vascular team then performed a second aortogram, which again demonstrated no extravasation of contrast or other abnormal finding. The remaining screws and rods were then removed through the posterior incision. A final aortogram after removal of all hardware showed no evidence of injury or abnormal flow within the thoracic aorta (-Fig. 3). Given the normal aortogram, it was decided no graft placement or further endovascular intervention was necessary.

The posterior wound was thoroughly irrigated and closed in layered fashion and the patient was returned to the supine position. The vascular catheters were removed and the groin incision was closed. Estimated blood loss for the procedure was $200 \mathrm{~mL}$. The patient was awakened and found to be neurologically and vascularly intact, unchanged from his preoperative examination. He was admitted to the surgical floor where he progressed appropriately and was discharged home on postoperative day 2 . He is now more than 2 years out from the procedure. He is pain free and has maintained a normal peripheral vascular examination. 


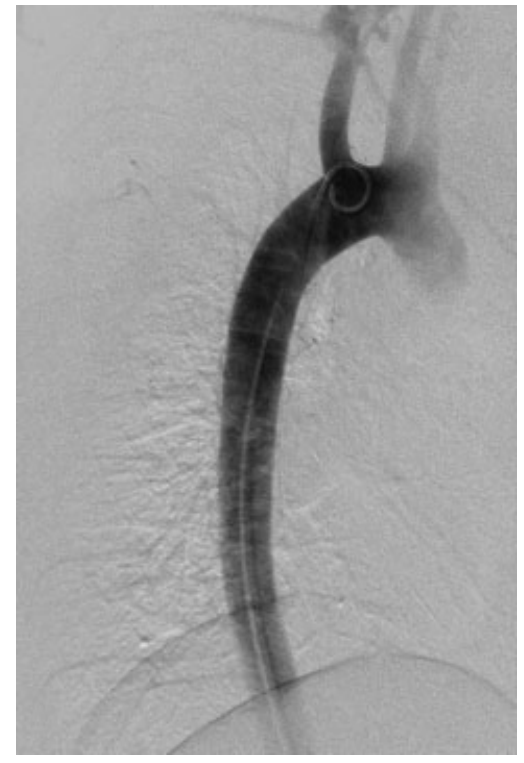

Fig. 3 Intraoperative aortography demonstrates no extravasation of contrast and normal filling of the descending thoracic aorta.

\section{Discussion}

Vascular injury has been recognized as a rare potential complication during anterior spine procedures, with a rate of less than $5 \%$ reported in a recent systematic review. ${ }^{11}$ After posteriorly based procedures, reported rates of acute vessel injury in systematic reviews range from 0 to $0.05 \%{ }^{2,3}$ However, because of the intimate association of the aorta with the thoracic and lumbar spine, it remains at risk of acute or delayed injury from misplaced posterior instrumentation. ${ }^{1}$ A growing number of reports suggest such injuries are likely an underappreciated complication. ${ }^{4-10}$

In cases of frank perforation of the aorta or another major vessel, immediate operative or endovascular intervention is indicated to repair the injury. However, optimal management of malpositioned instrumentation abutting the aorta is not well understood. Potential risks of impinging instrumentation include late catastrophic hemorrhage, pseudoaneurysm formation, or thromboembolic complications. These risks must be balanced against the surgical risk of removing such instrumentation. The best evidence in favor of observing impinging hardware without intervening comes from Foxx et al who retrospectively reviewed the position of 680 pedicle screws in 107 patients on routine postoperative imaging. Of the 680 screws, 33 screws (4.9\%) were found to contact a major vessel on imaging, including the aorta in four cases. Eight of the nine affected patients were living and asymptomatic at average follow-up of 25 months, with one patient deceased from amyotrophic lateral sclerosis at 16 months after surgery. ${ }^{12}$ From these data, the authors concluded that asymptomatic, impinging screws may be safely observed with serial imaging.

Evidence in favor of hardware removal comes primarily from case reports in which pedicle screws impinging against the aorta were diagnosed on a delayed basis and successfully managed with hardware removal and EVAR. ${ }^{4-8,10}$ Exact details of the procedures varied, but in all cases patients had endovascular stent grafts placed that bridged the affected region of the aorta before or after removal of their hardware. Stenting was advocated as a safer and less morbid alternative to open aortic repair. No complications were reported. ${ }^{4-8,10}$

Of note, in several of these reports, patients experienced a symptom-free period after their initial spine surgery and were diagnosed with impinging hardware after returning 4 months to 6 years later with new complaints of pain in the instrumented region; these complaints resolved after screw removal. ${ }^{6,8,10}$ We hypothesize that in the setting of impinging hardware, new-onset pain after a symptom-free period may potentially represent a "red-flag" for surgical intervention.

In an in vivo bovine model in which thoracic pedicle screws were left abutting the aorta, Faro et al found histopathologic changes in 96\% of aortic specimens at 3, 6, and 12 months. More than half of the specimens showed significant wall thinning, and in aggregate, the impinged aortas demonstrated decreased stress to failure compared with controls. The severity of tissue damage on histology did not correlate with the depth of impingement on CT scans. ${ }^{13}$ In the context of the limited clinical data, this study raises concerns that screw impingement may compromise of vessel wall integrity even in the absence of frank perforation.

The case considered here is unique in that our patient was much younger than those in previous reports ( 20 years old vs. older than 60 years) and because he was successfully managed without endovascular grafting given his benign findings on intraoperative aortography. As the incidence of pedicle screw instrumentation for AIS continues to rise, one expects to see younger patients facing decisions about how to manage impinging hardware. ${ }^{3}$ On the basis of multiple case reports, placement of an endovascular aortic graft appears to represent an effective, low-morbidity treatment for impinging or perforating pedicle screws in older population. However, available clinical data after EVAR in the vascular literature suggest a significant incidence of complication and reoperation at intermediate to long-term follow-up that would be more relevant to a younger patient. In one series of 1,066 patients treated with EVAR for abdominal aortic aneurysm and followed up between 2 and 16 years, 233 patients (22\%) required repeat operation, with 131 operations attributed to graft-related complications (12\%), and the remainder to progression of vascular disease. ${ }^{14}$ In another study using a modern endovascular aortic graft exclusively (Endurant; Medtronic, Minneapolis, Minnesota, United States), 16 of 100 grafts showed leakage on CT angiography and 5 of 100 patients required endovascular or operative reintervention within the first year. ${ }^{15}$

The high rate of complications and reoperation associated with EVAR is sobering, particularly when considering application in an adolescent patient. Here, we describe the safe and successful removal of a pedicle screw impinging on the aorta in a symptomatic 20 -year-old patient. On the basis of normal intraoperative aortography, we elected against placement of endovascular graft given the high incidence of associated 
complications and the expected longevity requirement in a young patient. At more than 2 years after the procedure, the patient remains asymptomatic with a normal vascular examination. We would recommend considering similar treatment of other young patients when intraoperative aortography shows no abnormality.

\section{Disclosures}

The study required no outside funding. The study authors have no financial interest in any of the products or techniques discussed.

\section{References}

1 Nathan H. Relations of the soft structures of the posterior mediastinum in the scoliotic spine. Acta Anat (Basel) 1988;133(3): 260-264

2 Papadoulas S, Konstantinou D, Kourea HP, Kritikos N, Haftouras N, Tsolakis JA. Vascular injury complicating lumbar disc surgery. A systematic review. Eur J Vasc Endovasc Surg 2002;24(3):189-195

3 Hicks JM, Singla A, Shen FH, Arlet V. Complications of pedicle screw fixation in scoliosis surgery: a systematic review. Spine 2010; 35(11):E465-E470

4 Kakkos SK, Shepard AD. Delayed presentation of aortic injury by pedicle screws: report of two cases and review of the literature. J Vasc Surg 2008;47(5):1074-1082

5 Minor ME, Morrissey NJ, Peress R, et al. Endovascular treatment of an iatrogenic thoracic aortic injury after spinal instrumentation: case report. J Vasc Surg 2004;39(4):893-896

\section{Commentary}

\author{
Gregory M. Malham ${ }^{1}$
}

\author{
${ }^{1}$ Neuroscience Clinical Institute, Epworth Hospital, Melbourne, \\ Victoria, Australia
}

Potter et al are to be commended for presenting an important yet very uncommon surgical complication of a misplaced thoracic screw requiring revision for aortic impingement. ${ }^{1}$ The index procedure was an excision of a T7 osteoblastoma 5 years previously. The presentation of the now 20 -year-old male patient was midthoracic and retrosternal pain without any evidence of tumor recurrence.

A computed tomographic (CT) thoracic scan performed to exclude change in instrumentation or pathology demonstrated a laterally misplaced left T6 screw. The screw position was stable and seemingly unchanged from the initial surgery. Interestingly, the CT demonstrated impingement on the thoracic aorta by the screw tip, which was previously unknown.

The lateral screw was unlikely to be the cause of the patient's pain. If pain is present, $12 \%$ of the patients will report improvement with removal of instrumentation ${ }^{2}$; this also facilitates future imaging for tumor surveillance.
6 Wegener B, Birkenmaier C, Fottner A, Jansson V, Dürr HR. Delayed perforation of the aorta by a thoracic pedicle screw. Eur Spine J 2008;17(Suppl 2):S351-S354

7 Clarke MJ, Guzzo J, Wolinsky JP, Gokaslan Z, Black JH III. Combined endovascular and neurosurgical approach to the removal of an intraaortic pedicle screw. J Neurosurg Spine 2011;15(5):550-554

8 Tschoeke SK, Gahr P, Krug L, Kasper AS, Heyde CE, Ertel W. Late diagnosis of pedicle screw malplacement with perforation of the thoracic aorta after posterior stabilization in a patient with multiple myeloma: case report. Spine 2011;36(13):E886-E890

9 Smythe WR, Carpenter JP. Upper abdominal aortic injury during spinal surgery. J Vasc Surg 1997;25(4):774-777

10 Loh SA, Maldonaldo TS, Rockman CB, et al. Endovascular solutions to arterial injury due to posterior spine surgery. J Vasc Surg 2012; 55(5):1477-1481

11 Wood KB, Devine J, Fischer D, Dettori JR, Janssen M. Vascular injury in elective anterior lumbosacral surgery. Spine 2010;35(9, Suppl): S66-S75

12 Foxx KC, Kwak RC, Latzman JM, Samadani U. A retrospective analysis of pedicle screws in contact with the great vessels. J Neurosurg Spine 2010;13(3):403-406

13 Faro FD, Farnsworth CL, Shapiro GS, et al. Thoracic vertebral screw impingement on the aorta in an in vivo bovine model. Spine 2005; 30(21):2406-2413

14 Quinney BE, Parmar GM, Nagre SB, et al. Long-term single institution comparison of endovascular aneurysm repair and open aortic aneurysm repair. J Vasc Surg 2011;54(6):1592-1597, discussion 1597-1598

15 van Keulen JW, de Vries JP, Dekker H, et al. One-year multicenter results of 100 abdominal aortic aneurysm patients treated with the Endurant stent graft. J Vasc Surg 2011;54(3):609-615
The T6 pedicles are among the most technically challenging to cannulate given their narrow width. Many surgeons perform plain radiographs to check spinal instrumentation position. Postoperative CT scans are equally used in many practices, including my own, to exclude neural and/or vascular compromise. A CT angiogram is very sensitive to both luminal and mural vascular abnormalities.

Delayed aortic rupture is extremely rare. ${ }^{3}$ However, removal of the $\mathrm{T} 6 \mathrm{screw}$ in this case for vascular impingement is reasonable, especially given the bovine in vivo studies of aortic wall thinning illustrated by Faro et al. ${ }^{4}$ This is especially true in a young adult. In the setting of luminal or gross mural abnormality, screw removal is mandatory but is likely to be more complicated.

The aorta undergoes constant cyclical movement with cardiac pulsatility being the primary dynamic elastic artery. With each cycle, it undergoes radial and longitudinal distortion. If the point of the screw was in contact with the aorta, it 
is unlikely that it would be in contact with a single adventitial point promoting erosion over time. ${ }^{4}$

The aorta is tethered to the vertebral column by multiple radicular branches; one is the artery of the Adamkiewicz, classically thought to arise on the left near the T9 level. Although the absence of erosion, thickening, or false aneurysm in the referable area is comforting, having an expedited means for brisk vascular control is vital. Combined management with the vascular surgery team involves preparing and positioning the patient for an immediate posterolateral thoracotomy (including a double lung intubation) as detailed by the authors. Endoluminal techniques permit control via a compliant balloon that can be prophylactically placed and repair subsequently effected with an appropriately sized stent graft. Ideally, imaging the descending thoracic aorta to position a wire and balloon opposite the screw and fix it relative to the sheath allows immediate control to be achieved.

A limitation of this case report is extrapolating results and complication rates from abdominal aortic aneurysm endovascular aortic repair (EVAR) to thoracic endovascular aortic repair (TEVAR) for trauma. Even for aneurysm repair, TEVAR has lower complication rates ${ }^{5}$ than the more complicated abdominal EVAR procedures. Furthermore, in this report, the injury is small and the thoracic aortic wall probably normal, and so seal is much more assured, provided proper planning has been performed, making an endoleak very unlikely. There is no aneurysm sac to pressurize in this setting that is the main source of reintervention in EVAR. EVAR results are not a surrogate for posttraumatic TEVAR results. Not placing the stent graft in the absence of an aortic abnormality is supported. The main concerns with placement of a stent graft in this report is the risk of paraplegia, the potential for devastating (but rare) graft infection, and the uncertainty of the long-term fate of the stent graft in young patients.

\section{References}

1 Heary RF, Bono CM, Black M. Thoracic pedicle screws: postoperative computerized tomography scanning assessment. J Neurosurg 2004;100(4, Suppl Spine):325-331

2 Stavridis SI, Bücking P, Schaeren S, Jeanneret B, Schnake KJ. Implant removal after posterior stabilization of the thoraco-lumbar spine. Arch Orthop Trauma Surg 2010;130(1):119-123

3 Kakkos SK, Shepard AD. Delayed presentation of aortic injury by pedicle screws: report of two cases and review of the literature. J Vasc Surg 2008;47(5):1074-1082

4 Faro FD, Farnsworth CL, Shapiro GS, et al. Thoracic vertebral screw impingement on the aorta in an in vivo bovine model. Spine (Phila Pa 1976) 2005;30(21):2406-2413

5 Marone EM, Kahlberg A, Tshomba Y, Chiesa R. Single-center experience with endovascular treatment of acute blunt thoracic aortic injuries. J Cardiovasc Surg (Torino) 2013;54(1):123-131

\section{Editorial Perspective}

The honest reporting of this complication by the authors deserves our respect. The post-discovery decision-making described here by the authors and the commenter is very helpful for all of us clinicians. This is a classic scenario for which there is no "evidence base" as we have no control group; we have to use our judgment, experience, and the few descriptions offered in the peer-reviewed literature, even if its level of evidence is 4 or 5 . This case raises the bigger question: Should all patients have some form of routine postinstrumentation advanced imaging to check on segmental hardware placement even in asymptomatic patients? What do you think? EBSJ invites you to share your thoughts. 